\title{
Preparation of Solid Dispersion for Ethenzamide-Carbopol and Theophylline-Carbopol Systems Using a Twin Screw Extruder
}

\author{
Masatoshi Ozawa, Koji Hasegawa, Yorinobu Yonezawa, and Hisakazu Sunada* \\ Faculty of Pharmacy, Meijo University; 150 Yagatoyama, Tempaku-ku, Nagoya 468-8503, Japan. \\ Received January 21, 2002; accepted March 8, 2002
}

\begin{abstract}
In the present study, we prepared solid dispersions of water-insoluble and soluble drugs (ethenzamide (ETZ) and theophylline (THEO)) by the twin screw extruder method, which made it possible to control both kneading and heating at the same time under the fusion point of each drug, using three types of the controlled-release high-molecular-weight substance Carbopol (CAR) as the carrier. The solid dispersions obtained were evaluated and compared with those prepared by the organic solvent method. These products showed significantly increased solubility of ETZ, but the solubility of THEO was reduced indicating that CAR slows the release of THEO. It is important not only to simply knead under high pressure but to select the optimal operation temperature to bring these drugs into a semi-fusion state. Solid dispersions obtained by this method showed X-ray diffraction and differential scanning calorimetry (DSC) patterns similar to those obtained by the organic solvent method indicating that the former can be used as a simple and effective method for preparation of solid dispersions.
\end{abstract}

Key words solid dispersion; Carbopol; twin screw extruder; ethenzamide; theophylline

The use of poorly soluble drugs has a number of drawbacks such as increasing the dosage, administration frequency and the resultant occurrence of side effects. Furthermore, as solubility is a rate-limiting factor for absorption in the human body, it is important to increase the solubility of a drug to improve its bioavailability. Various pharmaceutical approaches have been adopted to improve solubility using micelles and synthesis of soluble derivatives and salts.

The solid dispersion method, by which a drug is dispersed in a carrier to make it amorphous, is one of the pharmaceutical approaches most commonly employed to increase bioavailability of poorly soluble drugs. ${ }^{1)}$ Various methods for preparation of solid dispersions including coprecipitation, lyophilization, spray drying, solvent evaporation, fusion and powder mixing methods have been reported. ${ }^{2)}$ The solvent evaporation method and fusion method have a number of drawbacks, including residual organic solvent and decomposition of the drug. ${ }^{3,4)}$ A pressurization/kneading/extruding method using a twin screw extruder is one of the methods proposed for this purpose. ${ }^{5)}$ This extruder, originally designed as an extraction/casting device for polymer alloys in the plastic industry, ${ }^{6}$ is now used to process cereals and "functionalize" food materials such as tissue products from animal protein. ${ }^{7}$ This device has already been used successfully to prepare solid dispersions of nifedipine and hydroxypropylmethylcellulosephthalate, ${ }^{8)}$ and floating dosage forms of nicardipine hydrochloride and hydroxypropylmethylcellulose acetate succinate. ${ }^{9)}$

In the present study, the properties of a solid dispersion of ethenzamide (ETZ), which is poorly soluble in water, and that of theophylline (THEO), which is readily soluble in water, prepared using a twin screw extruder were compared with their physical mixtures and a solid dispersion obtained using organic solvents. The solid dispersions were prepared using 3 grades of Carbopol ${ }^{\circledR}$ (CAR), a cross-linked polymer of acrylic acid used as a drug release controlling carrier, ${ }^{10)}$ biological mucous membrane adherent, viscosity donor and emulsifier with the fishnet gel structure (CAR971P) and fuzzball gel structure (CAR974P and 934P) shown in Table
1. ${ }^{11)}$ The effects of temperature on the degree of crystallinity of drugs in solid dispersions were also evaluated.

\section{Experimental}

Materials The physical properties of $\mathrm{CAR}^{\circledR}$ (CBC Co., Ltd., BF Goodrich), ETZ (Yoshitomi Fine Chemicals Co., Ltd.) and THEO (Wako Pure Chemical Industries, Ltd.) are listed in Tables 1 and 2. All other reagents used were analytical grade and were obtained.

Twin Screw Extruder ${ }^{8}$ The twin screw extruder (KEX-25, Kurimoto, Ltd.) used in the present study consisted of a hopper, barrels, a die, a kneading screw, and heaters. The thread interval of the feed screw decreases from the hopper side to the die side. A physical mixture introduced into the hopper is carried forward by the feed screw, kneaded under high pressure by the kneading screw, and extruded from the die. Temperature inside the barrels can be accurately controlled from 30 to $300^{\circ} \mathrm{C}$ with 4 independent heaters, and water can be introduced from the liquid injection port if necessary.

Preparation of Materials. ${ }^{8)}$ Twin Screw Extruder Method ETZ or THEO was mixed with CAR at the ratios shown in Table 3 at $42 \mathrm{rpm}$ for 20 min using a V-type mixer (VM-5, Fuji Powdal Co., Ltd.). Mixtures were then treated with the twin screw extruder at a screw rotation rate of $100 \mathrm{rpm}$, powder supply rate of $10 \mathrm{~g} / \mathrm{min}$, water supply rate of $1 \mathrm{ml} / \mathrm{min}$, and a barrel temperature of $85^{\circ} \mathrm{C}$. Treated mixtures were dried for $10 \mathrm{~h}$ using a dryer (inside temperature: $50^{\circ} \mathrm{C}$ ), pulverized using a QUADRO COMIL (QC-197S, Powlex Co., Ltd.) equipped with a grater-type screen $(2.39 \mathrm{~mm} \phi)$, dried overnight in a dryer, pulverized again using an automatic mortar (Model ANM200 E, Nitto Kagaku Co., Ltd.), dried overnight again in a dryer to remove water completely, and sieved through a $150-250 \mu \mathrm{m}$ sieve to obtain a sample for granule dissolution. Samples $53 \mu \mathrm{m}$ or finer were used for powder X-ray diffraction and differential scanning calorimetric (DSC) analysis.

Mixtures of designated ratios were put into $50 \mathrm{ml}$ sample bottles and mixed for $5 \mathrm{~min}$ using a test tube shaker (Vortex-Genie-2, SM Kiki Co., Ltd.) to obtain physical mixtures.

Organic Solvent Method ETZ (50 g) was dissolved in $200-500 \mathrm{ml}$ of a 1:1 (w/w) mixture of ethanol and dichloromethane, and CAR $(50,150$, $250 \mathrm{~g}$ ) of 3 different grades was suspended in the resultant solutions. For establishment of equilibrium, the suspensions thus obtained were allowed to stand overnight at room temperature and pressure, and the solvent was spontaneously evaporated. The solvent was then evaporated under reduced pressure for $24 \mathrm{~h}$ at $60^{\circ} \mathrm{C}$ using a thermostatic reduced pressure dryer (Ishii Vacuum Sample Oven, Ishii Labo Works Co., Ltd.). The dry materials obtained after evaporation were pulverized, dried and sieved as described above and their physical properties were evaluated.

Evaluation of Solid Dispersions. Powder X-Ray Diffraction Powder $\mathrm{X}$-ray diffraction was conducted using a linear X-ray diffraction system (RAD-IIVC, Rigaku Denki Co., Ltd.) in which CuK $\alpha$-rays $(40 \mathrm{kV}, 20 \mathrm{~mA})$ were used as X-rays. The degree of diffraction was measured at $4 \%$ min every $0.02^{\circ}$ between 4 and $40^{\circ}(2 \theta)$. 
Table 1. Property of Carbopols and Its Gel Styructure

\begin{tabular}{|c|c|c|c|c|c|c|}
\hline $\begin{array}{l}\text { Polymer } \\
\text { (grade) }\end{array}$ & $\begin{array}{l}\text { Density } \\
\left(\mathrm{g} / \mathrm{cm}^{3}\right)\end{array}$ & $\mathrm{MW}^{a}$ & $\begin{array}{l}\text { Viscosity }^{b)} \\
\text { (CPS) }\end{array}$ & $\begin{array}{c}\text { Polymerization } \\
\text { solvent }\end{array}$ & \multicolumn{2}{|c|}{ Gel structure } \\
\hline CAR-971P & 1.41 & 1250000 & $4000-11000$ & Ethyl acetate & $\begin{array}{l:l} & \ddots \\
\ddots & \vdots\end{array}$ & $\begin{array}{c}\text { Fishnet } \\
\text { Carbopol 971P }\end{array}$ \\
\hline CAR-974P & 1.41 & 3000000 & $29400-39400$ & Ethyl acetate & & \\
\hline CAR-934P & 1.41 & 3000000 & $29400-39400$ & Benzene & $\begin{array}{l}6 \\
68\end{array}$ & $\begin{array}{c}\text { Fuzzball } \\
\text { Carbopol 974P/934P }\end{array}$ \\
\hline
\end{tabular}

a) Nominal-average molecular weight. b) $0.5 \%$ solution, $25^{\circ} \mathrm{C}, \mathrm{pH} 7.5$.

Table 2. Property of Drugs

\begin{tabular}{cccc}
\hline Drug & MW & Melting point $\left({ }^{\circ} \mathrm{C}\right)$ & Application \\
\hline Ethenzamide (ETZ) & 165.19 & Antipyretic agent \\
Theophylirheumatic agent \\
Anti-inflammatory
\end{tabular}

Table 3. Formulations of Physical Mixtures

\begin{tabular}{|c|c|c|c|c|c|c|c|c|c|c|c|}
\hline Ethenzamide (g) & 60 & 30 & 20 & 60 & 30 & 20 & 60 & 30 & 20 & - & - \\
\hline Theophylline (g) & - & - & - & - & - & - & - & - & - & 60 & 20 \\
\hline CAR-971P-NF (g) & 60 & 90 & 100 & - & - & - & - & - & - & 60 & 100 \\
\hline CAR-974P-NF (g) & - & - & - & 60 & 90 & 100 & - & - & - & - & - \\
\hline CAR-934P-NF (g) & - & - & - & - & - & - & 60 & 90 & 100 & - & - \\
\hline Total amount (g) & 120 & 120 & 120 & 120 & 120 & 120 & 120 & 120 & 120 & 120 & 120 \\
\hline
\end{tabular}

DSC Fusion temperature of each sample (10 mg) was measured at a temperature gradient of $10^{\circ} \mathrm{C} / \mathrm{min}$ in atmospheric air. A differential scanning calorimeter (DSC-3100, Mac Science Co., Ltd.) was employed.

Fourier Transformation IR Spectroscopy (FT-IR) Drug-carrier interactions in samples were determined based on IR spectra using a FT-IR spectroscope (FT-200, Horiba Ltd.) by the KBr method.

Dissolution Test The amount of drug released from samples was determined using a dissolution tester (NTR-3000, Toyama Sangyo Co., Ltd.) according to dissolution test method 2 (paddle method) described in the Japanese Pharmacopoeia (JP) XIII. The paddle was rotated at $100 \mathrm{rpm}$ using $50 \mathrm{mg}$ of treated powder and, as test fluid, $900 \mathrm{ml}$ of purified water adjusted to $37 \pm 0.2{ }^{\circ} \mathrm{C}$ and the 1 st and 2 nd fluids used in the disintegration test according to JP XIII. Test fluids (about $1 \mathrm{ml}$ ) were sampled at regular time intervals through a glass filter and immediately filtered through a $0.45 \mu \mathrm{m}$ membrane filter (DISMIC-25CP, Toyo Filter Paper Co., Ltd.). The amount of drug in the filtrate was assayed by HPLC (LC-9A, Shimadzu Corp.) under the following conditions: column, Shim-pack VP-ODS $(4.6 \times 150 \mathrm{~mm}$, $5 \mu \mathrm{m})$; mobile phase, a $6: 4(\mathrm{w} / \mathrm{w})$ mixture of methanol and purified water; flow rate, $1 \mathrm{ml} / \mathrm{min}$; wavelength, $293 \mathrm{~nm}$ for ETZ and $340 \mathrm{~nm}$ for THEO. A Chromatopack C-R4A (Shimadzu Corp.) was employed for data processing.

The Relationship between Operation Temperature and the Degree of Crystallinity A 1:1 mixture of ETZ and CAR-934P was treated by the twin screw extruder method at barrel temperatures ranging from 40 to $115^{\circ} \mathrm{C}$ to evaluate the effects of barrel temperature on the properties of extruder-treated samples. Integrated intensity of ETZ $\left(I_{\mathrm{ETZ}}\right)$ and solid dispersions of each temperature $\left(I_{\mathrm{SD}}\right)$ at $2 \theta\left(4-40^{\circ}\right)$ were determined from the diffraction intensity. The integrated intensity of CAR-934P treated with the twin screw extruder $\left(I_{\mathrm{CAR}}\right)$ was subtracted from $\left(I_{\mathrm{SD}}\right)$, and the ratio of the value obtained to the integrated intensity of ETZ was calculated as the degree of crystallinity at each temperature using Eq. 1.

degree of crystallinity $=\left(I_{\mathrm{SD}}-I_{\mathrm{CAR}}\right) / I_{\mathrm{ETZ}} \times 100$

\section{Results and Discussion}

Evaluation of Solid Dispersion. Changes in Crystallinity Powder X-ray diffraction patterns obtained with ETZ alone, CAR alone, their 1:1 physical mixture, and ETZCAR-P-NF treated by the twin screw extruder method and the organic solvent method are shown in Fig. 1, and that of THEO alone, a $1: 1$ physical mixture of THEO and CAR, and mixture of THEO and CAR treated by the twin screw extruder method are shown in Fig. 2.

ETZ alone showed marked crystallinity with many diffraction peaks. The high-molecular weight carrier CAR showed a broad diffraction pattern. The physical mixture showed ETZderived diffraction peaks. Solid dispersions prepared by the twin screw extruder method and the organic solvent method showed decreasing peak intensity as the ratio of CAR increased, with diffraction peaks showing almost same pattern of the organic solvent method at a ratio of $1: 5$ (ETZ:CAR) indicating that solid dispersion was formed. These diffraction patterns closely resembled the ratio of ETZ and CAR in each mixture.

THEO alone showed marked crystallinity with many diffraction peaks. The physical mixture also showed THEO-derived diffraction peaks. Solid dispersions prepared by the twin screw extruder method showed small peak intensity at a ratio of $1: 1$ (THEO:CAR). At a ratio of $1: 5$ (THEO: $\mathrm{CAR})$, refraction peaks disappearing completely, showing a 

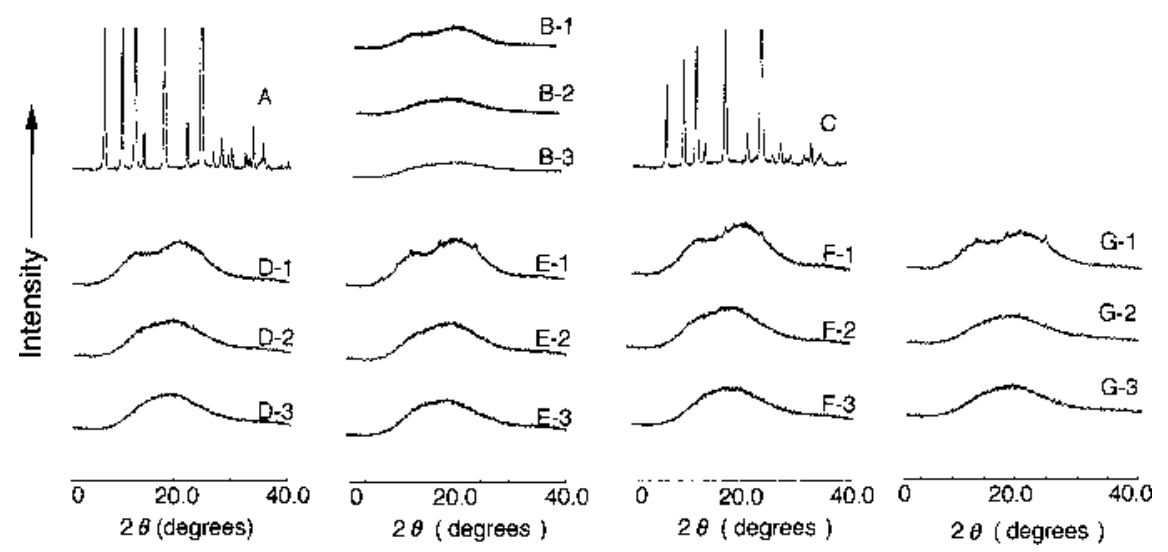

Fig. 1. Powder X-Ray Diffraction Patterns of ETZ, CAR, Physical Mixtures and Solid Dispersions Prepared by the Twin Screw Extruder Method

A) ETZ, B-1) CAR-971P, B-2) CAR-974P, B-3) CAR-934P, C) physical mixture (ETZ: CAR-971P=1:1). Twin screw extruder method, ETZ: CAR-971P, D-1) 1:1, D-2) $1: 3$, D-3) $1: 5$; twin screw extruder method, ETZ:CAR-974P, E-1) 1:1, E-2) $1: 3$, E-3) $1: 5$; twin screw extruder method, ETZ:CAR-934P, F-1) 1:1, F-2) $1: 3$, F-3) $1: 5$; organic solvent method, ETZ:CAR-971P, G-1) $1: 1$, G-2) $1: 3$, G-3) $1: 5$.

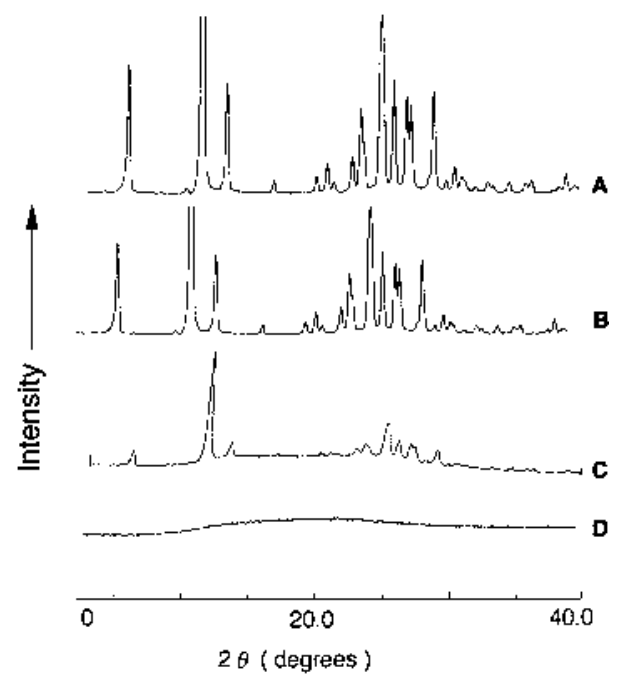

Fig. 2. Powder X-Ray Diffraction Patterns of THEO, Physical Mixtures and Solid Dispersions Prepared by the Twin Screw Extruder Method

A, THEO; B, physical mixtyure (THEO : CAR-971P=1:1); , twin screw extruder method (THEO : CAR-971P=1:1); $\mathrm{D}$, twin screw extruder method (THEO:CAR$971 \mathrm{P}=1: 5$ )

completely hollow pattern that of the solid dispersion.

In evaluation of solid dispersions prepared by the twin screw extruder method using CAR of different grades, CAR971P was found to give less diffraction peaks than CAR974P and CAR-934P at a mixing ratio of $1: 1$ (ETZ:CAR) indicating that CAR-971P have more ability to form the solid dispersion than others.

DSC Curves DSC curves of ETZ, CAR, their physical mixture, and solid dispersions obtained by the twin screw extruder method and the organic solvent method are shown in Fig. 3 and those of THEO, physical mixture of THEO and CAR, and solid dispersions obtained by the twin screw extruder method are shown in Fig. 4.

ETZ showed a sharp endothermic peak at $139^{\circ} \mathrm{C}$ due to fusion, while CAR showed a broad endothermic peak. The physical mixture showed an ETZ-derived endothermic peak.

Solid dispersions prepared by both the twin screw extruder method and the organic solvent method showed broad curves in the ETZ-derived endothermic point. No differences be- tween CAR of different grades were seen.

The observation that similar patterns were obtained with samples obtained by the twin screw extruder method and the organic solvent evaporation method confirmed that ETZ changed to an amorphous state in mixtures of ETZ and CAR and solid dispersions were formed when treated by the twin screw extruder method.

When the twin screw extruder method was employed, the solid dispersion prepared using CAR-971P showed least crystallinity, probably because CAR of this grade is less cross-linked than the two other grades when swollen, and for this reason the drug is more readily dispersed into macromolecules and undergoes reactions such as hydrogen bonding when treated by the twin screw extruder method. In contrast, CAR-934P and CAR-974P show a dense cross-linkage pattern when they come into contact with water, preventing the drug from penetrating deeply into the CAR structure and inhibiting reactions such as hydrogen bonding.

THEO showed a sharp endothermic peak at $275^{\circ} \mathrm{C}$ due to fusion. The physical mixture also showed a THEO-derived endothermic peak. The 1:1 (THEO:CAR) solid dispersion prepared by the twin screw extruder method showed a slight endothermic peak shifted to a lower temperature, but the $1: 5$ (THEO : CAR) solid dispersion showed a broad curve with no endothermic peak.

These findings indicated that the twin screw extruder method made THEO amorphous in THEO-CAR samples, as well as ETZ in ETZ-CAR samples, and formed solid dispersions.

Confirmation of Interactions by IR Spectrum FT-IR spectra obtained with samples prepared by the twin screw extruder method and the organic solvent method using CAR971P are shown in Fig. 5 and those obtained with THEO alone and samples prepared by the twin screw extruder method are shown in Fig. 6.

As ETZ has a primary alkylamide group, binding of its crystals is thought to be fortified by dimer chain association. ETZ and the physical mixture of ETZ and CAR-971P showed $\mathrm{N}-\mathrm{H}$ stretching vibrations at 3371 and $3178 \mathrm{~cm}^{-1}$ due to the primary amide of ETZ, while samples prepared by the twin screw extruder method and the organic solvent method showed a new peak at $3455 \mathrm{~cm}^{-1}$, but no more peaks 


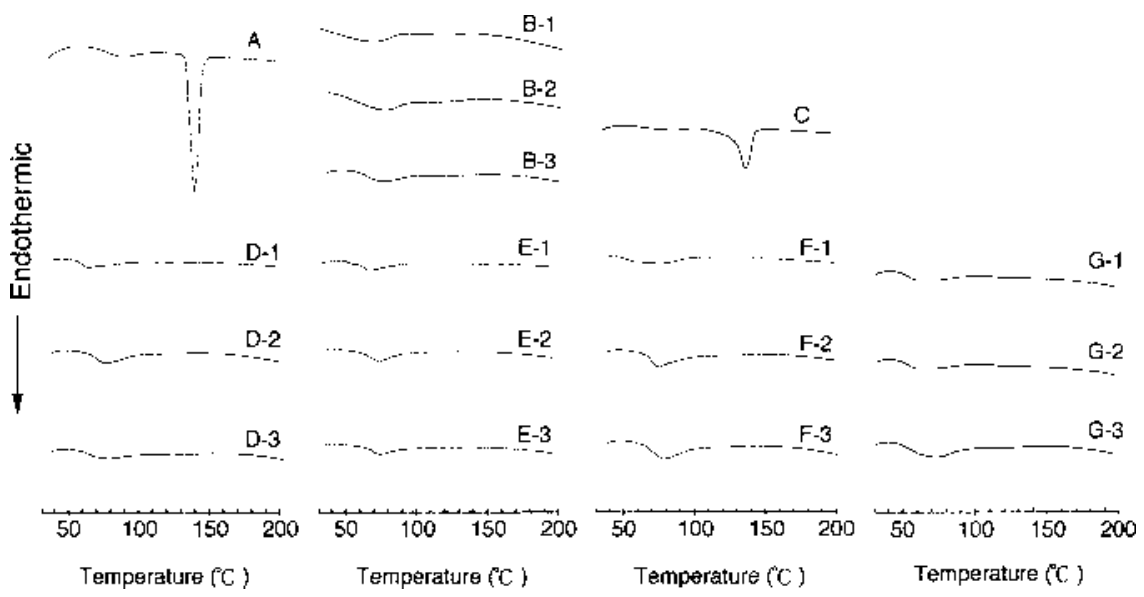

Fig. 3. Differential Scanning Calorimetry Curves of ETZ, CAR, Physical Mixtures and Solid Dispersions Prepared by the Twin Screw Extruder Method

A) ETZ, B-1) CAR-971P, B-2) CAR-974P, B-3) CAR-934P, C) physical mixture (ETZ:CAR-971P=1:1). Twin screw extruder method, ETZ: CAR-971P, D-1) 1:1, D-2) 1:3, D-3) 1:5; twin screw extruder method, ETZ:CAR-974P, E-1) 1:1, E-2) 1:3, E-3) 1:5; twin screw extruder method, ETZ:CAR-934P, F-1) 1:1, F-2) $1: 3$, F-3) $1: 5$; organic solvent method, ETZ:CAR-971P, G-1) 1:1, G-2) 1:3, G-3) $1: 5$.

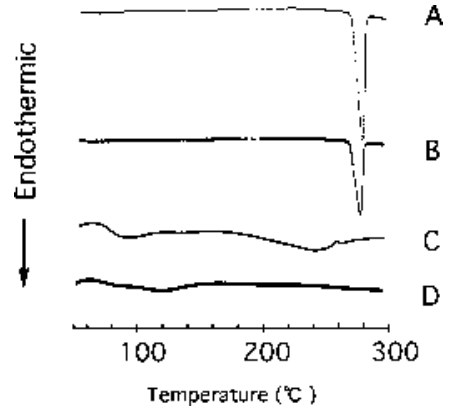

Fig. 4. Dofferential Scanning Calorimetry Curves of THEO, Physical Mixtures and Solid Dispersions Prepared by the Twin Screw Extruder Method

A) THEO, B) physical mixture (THEO : CAR-971P=1:1), C) twin screw extruder method (THEO:CAR-971P=1:1), D) twin screw extruder method (THEO:CAR$971 \mathrm{P}=1: 5$ ).

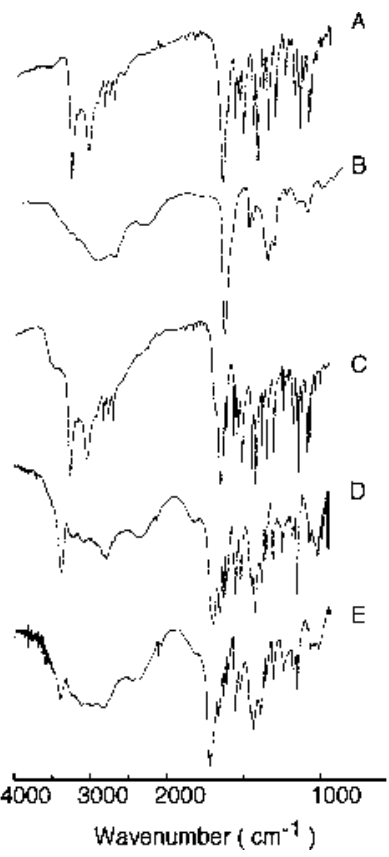

Fig. 5. IR Spectra of ETZ, CAR, Physical Mixtures and Solid Dispersions

A, ETZ; B, CAR-971P; C, physical mixture (ETZ: CAR-971P=1:1); D, twin screw extruder method (ETZ:CAR-971P=1:1); E, organic solvent method (ETZ:CAR$971 \mathrm{P}=1: 1)$.

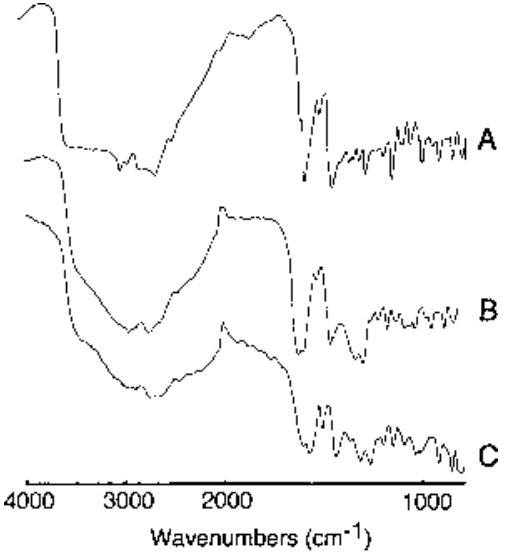

Fig. 6. IR Spectra of THEO, Physical Mixtures and Solid Dispersion Prepared by the Twin Screw Extruder Method

A, THEO; B, physical mixture (THEO : CAR-971P=1:1); , twin screw extruder method (THEO: CAR-971P=1:1).

due to primary amide at 3369 or $3176 \mathrm{~cm}^{-1}$. This was though to be because of interactions between the primary amide of ETZ and CAR during formation of the solid dispersion, which resulted in various associations such as hydrogen bonding between the $-\mathrm{NH}_{2}$-group of $\mathrm{ETZ}$ and $-\mathrm{COOH}$ group of CAR.

THEO alone and the physical mixture showed an $\mathrm{N}-\mathrm{H}$ deformation vibration at $1664 \mathrm{~cm}^{-1}$ due to the secondary amine of THEO, while samples prepared by the twin screw extruder method showed a shift of the $\mathrm{N}-\mathrm{H}$ deformation vibration to $1625 \mathrm{~cm}^{-1}$.

This finding suggested that the formation of solid dispersions of THEO and CAR by the twin screw extruder method was due to interactions of the two components and that hydrogen bonding between the $\mathrm{N}-\mathrm{H}$ of THEO and $\mathrm{COOH}$ of CAR is involved in these interactions.

Dissolution Test Dissolution profiles of ETZ from solid dispersions in purified water, the 1 st fluid and the 2 nd fluid are shown in Fig. 7 and those of THEO are shown in Fig. 8.

As a solid dispersion was fully formed when $1: 3$ (ETZ:CAR) and 1:5 (ETZ:CAR) ratios were used for the mixture, samples prepared by the twin screw extruder 

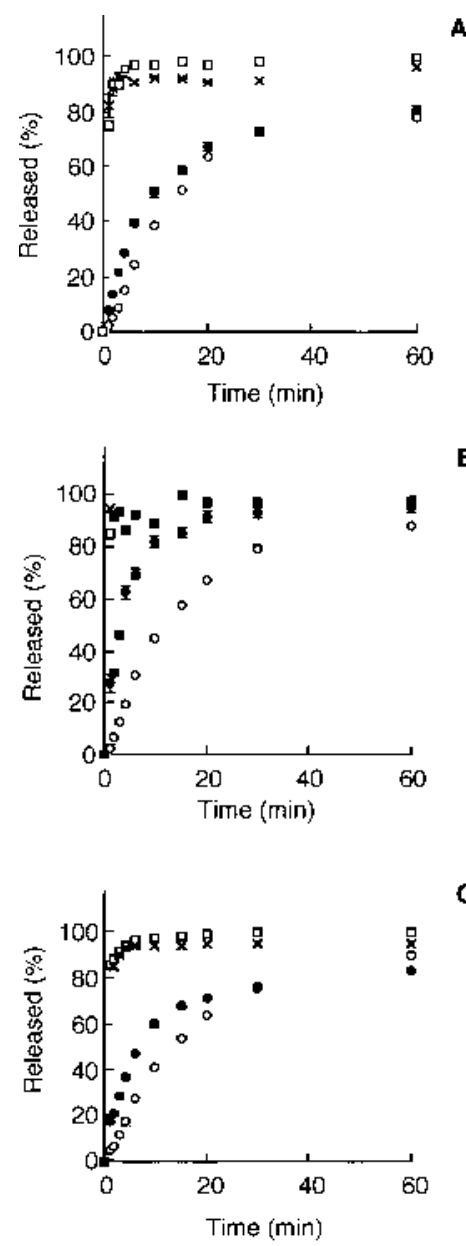

Fig. 7. Dissolution Profiles of ETZ from Solid Dispersions in $900 \mathrm{ml}$ at $37^{\circ} \mathrm{C}$ (CAR-971P)

A) water, B) 1st fluid, C) 2nd fluid. O, ETZ; $\bullet$, twin screw extruder method $(\mathrm{ETZ}: \mathrm{CAR}=1: 1) ; \times$, twin screw extruder method $(\mathrm{ETZ}: \mathrm{CAR}=1: 3) ; \square$, twin screw extruder method (ETZ: CAR $=1: 5)$. Each point represents the mean and S.D. $(n=3)$.

method showed more rapid dissolution than ETZ alone or the $1: 1$ (ETZ: CAR) mixture regardless of the CAR grade used. Slow dissolution, especially that observed in the $1: 1$ (ETZ:CAR) mixture in purified water and in the 2nd fluid compared to that seen in the 1st fluid, was thought to be because granules are more likely to adhere to each other immediately after the addition of samples to purified water and the 2nd fluid. Rapid dissolution as observed in the $1: 3$ and $1: 5$ (ETZ:CAR) mixture was thought to be because ETZ was dispersed completely and solid dispersions were formed. In the 1st fluid, granules were less likely to adhere to each other because CAR shows less swelling and the solubility of ETZ alone was higher at a low $\mathrm{pH}$, and this is thought to result in rapid dissolution. ${ }^{8)}$

To evaluate differences in the dissolution rate according to the grade of CAR, the times needed for $70 \%$ drug release in Figs. 7 and 8 were defined as the $\mathrm{T}_{70}$, and the relationship between $\mathrm{T}_{70}$ of ETZ and THEO, and CAR contents was evaluated (Figs. 9, 10).

When the CAR content was more than $50 \%$ rapid dissolution were observed, and no significant differences due to the CAR grade were observed in the 1 st fluid. In purified water and the 2nd fluid, however, marked differences were observed when the CAR content was $50 \%$. The total volume of
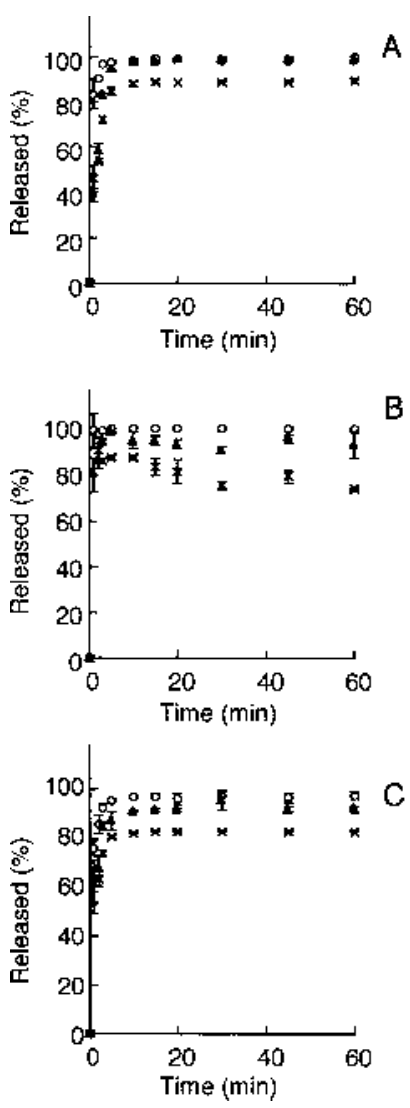

Fig. 8. Dissolution Profiles of THEO from Solid Dispersions in $900 \mathrm{ml}$ of at $37^{\circ} \mathrm{C}$ (CAR-971P)

A) water, B) 1st fluid, C) 2nd fluid. $\bigcirc$, THEO; $\times$, twin screw extruder method $($ THEO $:$ CAR $=1: 1) ; \boldsymbol{\Delta}$, twin screw extruder method (THEO $:$ CAR =1:5). Each point represents the mean and S.D. $(n=3)$.

expanded channel in the purified water, 1 st and 2 nd fluid differed in the structure differences of CAR grades which affected dissolution rate.

In neutral and alkaline solutions such as purified water and the 2 nd fluid, only samples with a 50\% CAR concentration show marked adhesion of granules during dissolution. In the case of CAR-974P, which is a semi-enteric diluent with a high degree of cross-linking (Table 1), which becomes completely hydrated at a high $\mathrm{pH}$, and forms a gelatin-like hydrogel layer, ETZ was released rapidly as a result of channel opening. CAR-934P showed a degree of cross-linking similar to that of CAR-974P, but it released the drug more slowly than CAR-974P due to poor dispersibility of the drug in the hydrogel layer. The drug release from CAR-971P was slowest because of thick hydrogel layer caused by the lowest degree of cross-linkage and the smallest number of channels generated by swelling.

As THEO is a soluble drug, it dissolved most rapidly when tested alone, and the release from samples treated by the twin screw extruder method was delayed due to CAR. As described above due to the interactions between the $\mathrm{N}-\mathrm{H}$ of THEO and $\mathrm{COOH}$ of CAR' released the drug more slowly and in some cases decreased because re-entrapped by CAR.

Relationship between Operation Temperature and the Degree of Crystallinity The degree of crystallinity at each temperature is shown in Fig. 11. The degree of crystallinity of ETZ decreased as barrel temperature increased, suggesting that when preparing solid dispersions of ETZ and CAR using 

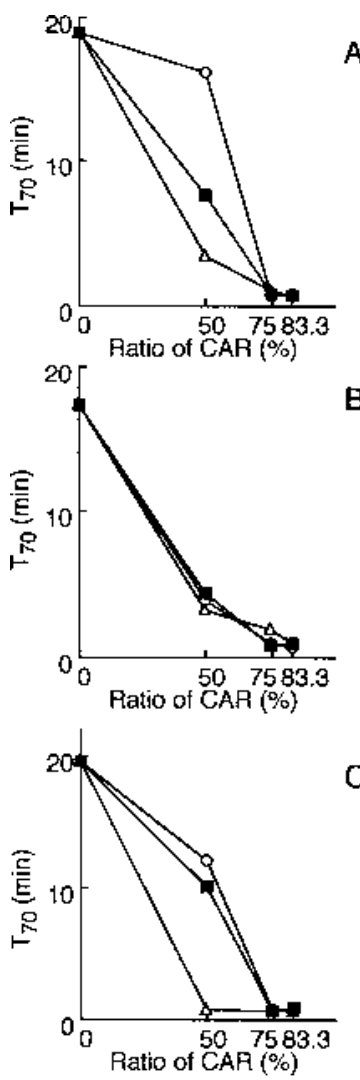

Fig. 9. Relationship between $\mathrm{T}_{70}$ of ETZ and Ratio of CAR A) water, B) 1st fluid, C) 2nd fluid. $\bigcirc$, CAR-971P; $\triangle$, CAR-974P; $\mathbf{\square}$, CAR-934P

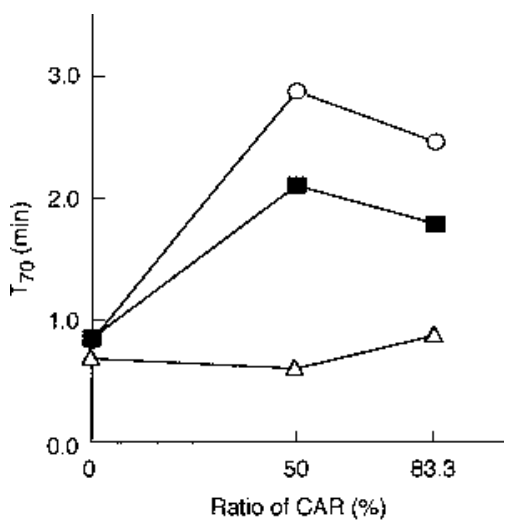

Fig. 10. Relationship between $\mathrm{T}_{70}$ of THEO and Ratio of CAR $\bigcirc$, water; $\triangle$, the 1 st fluid; $\mathbf{\square}$, the 2nd fluid.

a twin screw extruder, it is important not only to conduct kneading under high pressure but also to determine the temperature at which the drug is forms a semi-fused state. It is critical to select optimal operation conditions because excessively high temperature may cause decomposition of the

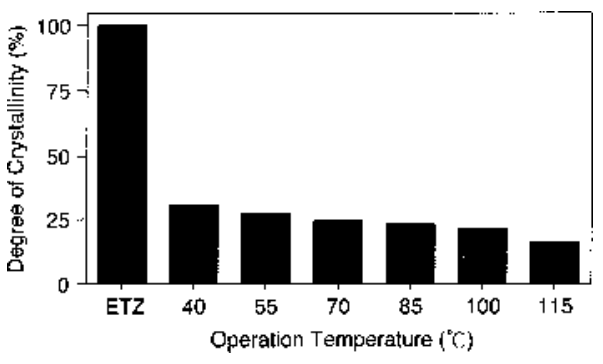

Fig. 11. Degree of Crystallinity of ETZ in Solid Dispersions Prepared at Various Temperatures

drug.

\section{Conclusions}

(1) Powder X-ray diffraction and DSC evaluation showed that when ETZ-CAR and THEO-CAR mixtures are treated with a twin screw extruder, ETZ and THEO are made amorphous and solid dispersions can be formed as by the organic solvent method.

(2) When preparing ETZ-CAR solid dispersions by the twin screw extruder method, it is important not only to simply knead under high pressure but to select optimal operation temperature to bring the drug into a semi-fusion state.

(3) Solid dispersions obtained using the twin screw extruder showed significantly increased ETZ release, but the release rate of THEO deceased when the same treatment was conducted indicating that $\mathrm{CAR}$ slows the release rate of THEO.

(4) Solid dispersions obtained by the twin screw extruder method and those obtained by the organic solvent method showed similar X-ray diffraction and DSC patterns, indicating that the former can be used as a simple and effective method for preparation of solid dispersions.

Acknowledgements The author is grateful to Kenichi Harada, Professor, and Kiyonaga Fujii, Research Associate, of the Department of Instrumental Analysis, for their help in the determination of FT-IR spectra.

References

1) Chiou W. L., Riegelman S., J. Pharm. Sci., 60, 1281-1302 (1971).

2) Sugimoto I., Inphachem, 2, 17-26, (1981).

3) Ford J. L., Stewart A. F., Rubinstein M. K., J. Pharm. Pharmacol., 31, $726-729$ (1979).

4) Hasegawa A., Sugimoto I., Kagaku Kogyo, 37, 309-317 (1986).

5) McGinity J. W., Zhang F., Repka M. A., Koleng J. J., Matsuda Y., Pharm. Tech. Jpn., 16, 897-913 (2000).

6) Hayakawa I., "Extrusion Cooking," KORIN, Tokyo, 1987, pp. 11-43.

7) Sakai T., High Polymers Jpn., 42, 768-771 (1993).

8) Nakamichi T., Urayasu H., Fukui H., Oka M., Izumi S., Ando T., Shimizu N., Ushimaru K., J. Pharm. Sci. Technol., 56, 15-22 (1996).

9) Nakamichi H., Yasuura H., Fukui H., Oka M., Izumi S., Int. J. Pharm., 218, 103-112 (2001).

10) Ozeki T., Yuasa H., Kanaya Y., J. Control. Res., 63, 287-295 (2000).

11) CBC Co., Ltd., Bulletin "Polymer for Pharmaceutical Application" CBC Co., Ltd. 1999. 